$\mathrm{DTP} / 97 / 16$

$\mathrm{ETH}-\mathrm{TH} / 97-10$

March 1997

\title{
QCD Corrections and the Leptoquark Interpretation of the HERA High- $Q^{2}$ Events
}

\author{
Z. Kunszt ${ }^{a}$ and W. J. Stirling ${ }^{b, c}$ \\ a) Institute of Theoretical Physics, ETH, Zürich, Switzerland. \\ b) Department of Physics, University of Durham, \\ c) Department of Mathematical Sciences, University of Durham, \\ South Road, Durham DH1 3LE, United Kingdom.
}

\begin{abstract}
The excess of high- $Q^{2}$ events found by $\mathrm{H} 1$ and ZEUS at HERA in $e^{+} p$ deep-inelastic scattering above the Standard Model prediction motivates us to calculate the NLO QCD corrections to the HERA scalar leptoquark (or squark) production cross sections. We find that the corrections are significant, of order $50 \%$ in the mass range of interest. We also calculate the leptoquark average transverse momentum squared and find it to be rather small. Various leptoquark production cross sections at the Tevatron $p \bar{p}$ collider are also considered. We investigate in detail the leptoquark interpretation of the HERA data. First we assume a minimal leptoquark model with a single diagonal Yukawa coupling to first family lepton and quark mass eigenstates only. In this case constraints from atomic parity violating experiments allow only isodoublet scalar leptoquark production at HERA. This interpretation can be confirmed or ruled out in the near future by high luminosity data at the Tevatron. The Tevatron data already appear to rule out the vector leptoquark interpretation of the HERA data. We also consider a more general model which allows for all possible left-handed, right-handed, flavour and lepton number changing couplings. The allowed values of the Yukawa couplings of this general model offer several different interpretations of the data which are radically different from the minimal model solutions. However these somewhat ad hoc tuned solutions can easily be tested by future HERA experiments.
\end{abstract}

\footnotetext{
${ }^{1}$ E-mails: kunszt@itp.phys.ethz.ch; W.J.Stirling@durham.ac.uk.
} 


\section{Introduction}

Recently the HERA collaborations H1 [1] and ZEUS [2] have presented new results on high- $Q^{2}$ deep inelastic $e^{+} p$ scattering based on $14 \mathrm{pb}^{-1}$ and $20 \mathrm{pb}^{-1}$ luminosity data, respectively. The data indicate an excess of events with respect to the Standard Model expectation predicted by the conventional next-to-leading order QCD-improved parton model in a new kinematical range unexplored by previous experiments. At high $Q^{2}$ values $Q^{2}>15000 \mathrm{GeV}^{2} \mathrm{H} 1$ finds 7 excess events while ZEUS finds 5 events. An interesting feature of the $\mathrm{H} 1$ events is that they are clustered in a single bin of the invariant mass of the jet-lepton system $\left(M_{\mathrm{ej}}=\sqrt{x s}\right)$ around $200 \mathrm{GeV}$ with an estimated experimental resolution of $6 \mathrm{GeV}$ [1]. The ZEUS events do not exhibit such a clustering, but this is not in conflict with the $\mathrm{H} 1$ data since ZEUS have a larger $M_{\mathrm{ej}}$ resolution. $\mathrm{H} 1$ have also published results on charged current events [1]. Although they find more events than expected, the excess is not statistically significant. If the signal is eventually confirmed as the production of a new heavy resonance, one will presumably be able to measure its spin and its branching ratios both to charged current and to flavour and/or lepton number violating channels.

The possibility of $s$-channel resonance production at HERA has been suggested in two theoretical schemes. First, Buchmüller and Wyler [3] argued that the leptoquarks (LQ) necessarily appearing in grand unified theories [4, 5] can also be accommodated in theories with conserved lepton and baryon number. They pointed out that these leptoquarks can be light (i.e. $m_{\mathrm{LQ}} \ll M_{\mathrm{GUT}}$ ) since low energy experiments impose much weaker bounds on lepton and baryon number conserving couplings. Second, the squarks $(\tilde{q})$ of supersymmetric extensions of the Standard Model can have direct (lepton number violating) couplings to quarks and leptons if $\mathrm{R}$-parity is not conserved. Therefore they can also be coupled to lepton-quark initial states and thus appear as $s$-channel resonances [6]. In the present study we shall restrict our discussion to the leptoquark interpretation only, although several of our results will remain valid also for squark production.

In section 2 we consider leptoquark production cross sections at HERA, including nextto-leading order QCD corrections. From the number of excess events we extract the value of the leptoquark couplings $(\lambda)$ and discuss the constraints on these coming from atomic parity violating experiments. We also comment on non-minimal models which allow for all possible Yukawa couplings, following the analysis of ref. [7].

In section 3 we consider single and pair production of scalar and vector leptoquarks in $p \bar{p}$ collisions at the Tevatron. We study $\lambda$ dependent contributions, the effects of QCD 
corrections, signals and backgrounds, and experimental limits on the leptoquark masses obtained by D0 and CDF.

Section 4 describes the calculation of the next-to-leading order QCD corrections to colour-triplet scalar resonance production in $e p$ collisions. The numerical value of the ' $K$-factor' and the average transverse momentum squared of the produced resonance are also studied. Full details of the calculations are given in the Appendix. Our conclusions are contained in section 5 .

\section{Leptoquark production at HERA and limits on couplings}

We first consider the minimal scheme in which the leptoquarks have separate baryon and lepton number conserving chiral couplings for each family of mass eigenstates. This allows us to evade severe low energy limits on lepton number violating couplings [7, 8, 9]. Leptoquarks which are coupled to the first family only are called first generation leptoquarks. A leptoquark is said to couple chirally if it couples either to left-handed (L) or right-handed $(\mathrm{R})$ leptons but not both.

The allowed leptoquark representations have been classified in ref. [8]. There are seven $B$ and $L$ conserving and $\mathrm{SU}(3) \times \mathrm{SU}(2) \times \mathrm{U}(1)$ renormalizable couplings both for scalar leptoquarks

$$
\begin{aligned}
\mathcal{L}_{S} & =g_{1 L} \bar{q}_{L}^{c} i \tau_{2} l_{L} S_{1}+g_{1 R} \bar{u}_{R}^{c} i \tau_{2} e_{R} S_{1}+\tilde{g}_{1 R} \bar{d}_{R}^{c} i \tau_{2} e_{R} \tilde{S}_{1}+g_{2 R} \bar{u}_{R} l_{L} S_{2} \\
& +g_{2 L} \bar{q}_{L} e_{L} S_{2}+\tilde{g}_{2 L} \bar{d}_{R} l_{L} \tilde{S}_{2}+g_{3 L} \bar{q}_{L}^{c} i \tau_{2} \vec{\tau} l_{L} S_{3}+\text { h.c. }
\end{aligned}
$$

and vector leptoquarks

$$
\begin{aligned}
\mathcal{L}_{V} & =h_{1 L} \bar{q}_{L} \gamma_{\mu} l_{L} V_{1}^{\mu}+h_{1 R} \bar{u}_{R} \gamma_{\mu} e_{R} V_{1}^{\mu}+\tilde{h}_{1 R} \bar{u}_{R} \gamma_{\mu} e_{R} \tilde{V}_{1}^{\mu}+h_{2 R} \bar{d}_{R}^{c} i \gamma_{\mu} l_{L} V_{2}^{\mu} \\
& +h_{2 L} \bar{q}_{L}^{c} \gamma_{\mu} e_{R} V_{2}^{\mu}+\tilde{h}_{2 L} \bar{u}_{R}^{c} \gamma_{\mu} l_{L} \tilde{V}_{2}^{\mu}+h_{3 L} \bar{q}_{L} \gamma_{\mu} \vec{\tau} l_{L} V_{3}^{\mu}+\text { h.c. }
\end{aligned}
$$

We can distinguish the leptoquarks according to their weak isospin properties: singlets (e.g. $\left.S_{1}\right)$, doublets $\left(S_{2}\right)$ and triplets $\left(S_{3}\right)$. The doublet scalar leptoquarks and the singlet and triplet vector leptoquarks have zero fermion number $(L+3 B)$ while the singlet and triplet scalar leptoquark and doublet vector leptoquark have fermion number two. Couplings are designated left or right handed according to the handedness of the lepton. 
We emphasize a difference in the theoretical status of scalar and vector leptoquarks. Within the Standard Model, vector leptoquarks cannot be considered as gauge bosons and therefore their couplings to the gauge bosons of the Standard Model are not renormalizable in general. Scalar leptoquarks, however, can be considered as possible renormalizable matter fields of the Standard Model. In particular, the squarks of the supersymmetric standard model with $\mathrm{R}$-parity violation provide a theoretically appealing realization of the isospin doublet and singlet leptoquarks $\left(\tilde{S}_{2}\right.$ and $\left.S_{1}\right)$. In contrast, scalar leptoquarks of exotic electric charge $\left(\tilde{S}_{1}\right)$, and $S_{3}$ and vector leptoquarks have no counterparts in supersymmetric models. In section 3 it will be shown that the Tevatron limits on the production of vector leptoquarks are only very marginally consistent with the vector leptoquark interpretation of the HERA events. In this section, therefore, we shall consider limits and production rates only for scalar leptoquarks.

The Yukawa couplings in eq. (11) are diagonal in the family number. In order to avoid the severe low energy limits from the leptonic decays of charged pions it is convenient to require that the leptoquarks have only one non-zero chiral coupling. Thus we can have left handed or right handed scalar leptoquarks $S_{1 R}, S_{1 L}$ and $S_{2 L}, S_{2 R}$ in (11). In Table 1 we list all possible scalar leptoquark states which can couple to a $e^{+} q$ initial state.

The leading order production cross section at HERA has the simple form (see Appendix A.1)

$$
\sigma=\frac{\pi \lambda^{2}}{4 s} q\left(m_{\mathrm{LQ}}^{2} / s\right) \cdot \mathrm{BR},
$$

where $\lambda^{2}$ denotes generically the coupling constants squared $\left(g_{L, R}^{2}\right)$ of the produced scalar leptoquark as listed in Table 1 (or two times the coupling constants squared $\left(h_{L, R}^{2}\right)$ of the vector leptoquarks). The cross sections of scalar leptoquark production calculated to nextto-leading order $\left(\mathcal{O}\left(\alpha_{S}\right)\right)$ accuracy are plotted in Fig. 1 as a function of the leptoquark mass $m_{\mathrm{LQ}}$ for the $e^{+} u, e^{+} d, e^{+} s, e^{+} \bar{u}$ and $e^{+} \bar{d}$ initial states' assuming $\lambda^{2}=1$. For other values of $\lambda$ the cross section obviously scales as $\lambda^{2}$. The cross section values vary strongly according to the initial quark flavour. For $m_{\mathrm{LQ}}=200 \mathrm{GeV}$ we find the ratios (see section 4 , Table 3)

$$
\sigma_{u}: \sigma_{d}: \sigma_{\bar{d}}: \sigma_{\bar{u}}: \sigma_{s}=1440: 352: 16.7: 7.32: 8.21
$$

where the values are given in picobarns. Assuming $80 \%$ experimental efficiency, we can extract from the H1 and ZEUS results a rough estimate for the HERA leptoquark production cross section of $0.7 \mathrm{pb}$. Comparing this value with the predictions of Fig. 1 for

${ }^{2}$ At $\mathcal{O}\left(\alpha_{S}\right)$ the $e^{+} g$ scattering process also contributes, see Appendix, and is included in the cross sections in Fig. 1. 


\begin{tabular}{|ll|c|c|c|c|}
\hline process & & $\lambda^{2}(\sigma)$ & HERA $\left(g^{2}\right)$ & APV $\left(g^{2}\right)$ & BR \\
\hline$e^{+} u \rightarrow S_{2}^{5 / 3} \rightarrow e^{+} u$ & $g_{2 R}^{2}(+) g_{2 L}^{2}$ & 0.00049 & $<0.024$ & 1 \\
$e^{+} d \rightarrow S_{2}^{2 / 3} \rightarrow e^{+} d$ & $g_{2 R}^{2}$ & 0.0020 & $<0.024$ & $\frac{g_{2 R}^{2}}{g_{2 R}^{2}+g_{2 L}^{2}} \rightarrow 1$ \\
& $\rightarrow \bar{\nu} u$ & $g_{2 L}^{2}$ & & - & $\frac{g_{2 L}^{2}}{g_{2 R}^{2}+g_{2 L}^{2}} \rightarrow 0$ \\
$e^{+} d \rightarrow \tilde{S}_{2}^{2 / 3} \rightarrow e^{+} d$ & $\tilde{g}_{2 L}^{2}$ & 0.0020 & $<0.024$ & 1 \\
$e^{+} \bar{u} \rightarrow S_{1}^{1 / 3}$ & $\rightarrow e^{+} \bar{u}$ & $g_{1 R}^{2}(+) g_{1 L}^{2}$ & $0.19,0.096$ & $<0.012$ & $\frac{g_{1 R}^{2}+g_{1 L}^{2}}{g_{1 R}^{2}+2 g_{1 L}^{2}} \rightarrow \frac{1}{2}, 1$ \\
& $\rightarrow \bar{\nu}$ & $g_{1 L}^{2}$ & & - & $\frac{g_{1 L}^{2}}{g_{1 R}^{2}+2 g_{1 L}^{2}} \rightarrow \frac{1}{2}, 0$ \\
$e^{+} \bar{d} \rightarrow \tilde{S}_{1}^{4 / 3} \rightarrow e^{+} \bar{d}$ & $\tilde{g}_{1 R}^{2}$ & 0.0420 & $<0.024$ & 1 \\
$e^{+} \bar{u} \rightarrow S_{3}^{1 / 3}$ & $\rightarrow e^{+} \bar{u}$ & $g_{3 L}^{2}$ & 0.096 & $<0.024$ & $\frac{1}{2}$ \\
& $\rightarrow \bar{\nu}$ & $g_{3 L}^{2}$ & & & $\frac{1}{2}$ \\
$e^{+} \bar{d} \rightarrow S_{3}^{4 / 3}$ & $\rightarrow e^{+} \bar{d}$ & $2 g_{3 L}^{2}$ & 0.021 & - & 1 \\
\hline
\end{tabular}

Table 1: Scenarios for scalar leptoquark production in $e^{+} p$ collisions, with values and limits for the various couplings obtained from HERA and atomic parity violation (APV). Limiting values for the branching ratios corresponding to definite chiral couplings (i.e. L or $\mathrm{R}$ ) are given.

$m_{\mathrm{LQ}}=200 \mathrm{GeV}$ we can calculate numerical values for the various coupling constants listed in Table 1, assuming branching ratios which correspond to definite chiral couplings.

In the the minimal scheme the strict bounds on electron and muon number conservation are automatically satisfied. Allowing only one chiral coupling, the severe constraints from the leptonic decays of charged pions and kaons are also avoided. But in the case of first generation leptoquarks the diagonal flavour conserving couplings (see Table 1) are also severely limited by the precise measurements of the atomic parity violating neutral current weak charge of the caesium atom

$$
Q_{W}=-376 C_{1 u}-422 C_{1 d}
$$

where $C_{1 u}$ and $C_{1 d}$ denote the lepton axial vector - quark vector interference terms of the effective low energy four fermion interaction

$$
\mathcal{L}_{\mathrm{APV}}=\frac{G}{\sqrt{2}} \sum_{i=u, d} C_{1 i} \bar{l} \gamma^{\mu} \gamma^{5} l \bar{q}_{i} \gamma_{\mu} q_{i}
$$

with

$$
C_{1 u}=-\frac{1}{2}+\frac{4}{3} \sin ^{2} \Theta_{W}, \quad C_{1 d}=\frac{1}{2}-\frac{2}{3} \sin ^{2} \Theta_{W}
$$


The scalar leptoquark contribution to $C_{1 q}$ is

$$
\Delta C_{1 q}=\frac{\sqrt{2} g^{2}}{8 G_{F} m_{\mathrm{LQ}}^{2}} .
$$

The measured value is 10

$$
Q_{W}=-71.04 \pm 1.58 \pm[0.88]_{\mathrm{th}}
$$

to be compared with the Standard Model prediction [10]

$$
Q_{W}=-72.88 \pm 0.05 \pm 0.03
$$

The difference between theory and experiment is therefore smaller than

$$
\left|\Delta Q_{W}\right|=3.6
$$

which gives

$$
g^{2}<0.024\left(\frac{m_{\mathrm{LQ}}}{200 \mathrm{GeV}}\right)^{2}
$$

which in turn leads to the values given in the fourth column of Table 1. From this we conclude that the HERA and the APV data are consistent only with the production of isodoublet (zero fermion number) leptoquarks. The parton distributions for antiquarks are too small at large $x(\sim 1 / 2)$ to allow sizeable production of leptoquarks with the weak coupling required by atomic parity violation data. If this interpretation is correct and only one leptoquark exists in the HERA kinematic range, then it follows that in $e^{-} p$ collisions the signal will be weaker by about two orders of magnitude! While this is an attractive explanation by itself, it may be excluded soon by new analyses from CDF and D0 at the Tevatron. We shall see in the next section that scalar leptoquarks of mass $m_{\mathrm{LQ}} \sim 200 \mathrm{GeV}$ are very close to the published discovery limit for leptoquark pair production at the Tevatron.

The Tevatron limit can be weakened by suppressing the branching ratio into charged lepton + jet, since the production is essentially independent of the leptoquark coupling. This motivates us to consider the more general parametrization of leptoquark production as studied by Davidson et al. [7], with general mixed couplings between the three families. In the Lagrangian $\mathcal{L}_{S}$ of (11) the family diagonal leptoquark couplings $g$ are replaced by the coupling matrix $g^{i j}$ where the summation labels run over the family indices $i, j=1,2,3$. Davidson et al. [7] summarize their results in terms of bounds on coupling constant

\footnotetext{
${ }^{3}$ See Table 15 of ref. [7].
} 


\begin{tabular}{|c|c|c|c|c|c|c|c|}
\hline$g^{1 j} g^{m n}$ & $S_{1 L}$ & $S_{1 R}$ & $\tilde{S}_{1 R}$ & $S_{2 L}$ & $S_{2 R}$ & $\tilde{S}_{2 L}$ & $S_{3 L}$ \\
\hline$(11)(11)$ & .006 & .008 & .008 & .008 & .004 & .008 & .004 \\
$(11)(12)$ & $4 \times 10^{-5}$ & .012 & $6 \times 10^{-4}$ & 0.012 & $6 \times 10^{-4}$ & $4 \times 10^{-5}$ & $4 \times 10^{-5}$ \\
$(11)(13)$ & .008 & $*$ & .012 & $*$ & .012 & .012 & .008 \\
$(11)(21)$ & $2 \times 10^{-6}$ & $2 \times 10^{-6}$ & $2 \times 10^{-6}$ & $2 \times 10^{-6}$ & $2 \times 10^{-6}$ & $2 \times 10^{-6}$ & $6 \times 10^{-7}$ \\
$(11)(23)$ & .008 & $*$ & .012 & $*$ & .012 & .012 & .008 \\
$(11)(31)$ & .006 & $2 \times 10^{-2}$ & $2 \times 10^{-2}$ & $2 \times 10^{-2}$ & $2 \times 10^{-2}$ & $2 \times 10^{-2}$ & .006 \\
$(11)(32)$ & $4 \times 10^{-5}$ & & .1 & & .1 & $4 \times 10^{-5}$ & $4 \times 10^{-5}$ \\
$(11)(33)$ & .008 & $*$ & .16 & $*$ & .16 & $.16^{-0}$ & .008 \\
$(12)(12)$ & 1.2 & & & & & & 1.2 \\
$(12)(13)$ & .08 & & $1.2 \times 10^{-3}$ & & $1.2 \times 10^{-3}$ & $1.2 \times 10^{-3}$ & $6 \times 10^{-4}$ \\
$(12)(21)$ & $4 \times 10^{-5}$ & .2 & $2 \times 10^{-6}$ & .2 & $2 \times 10^{-6}$ & $2 \times 10^{-6}$ & $1.2 \times 10^{-6}$ \\
$(12)(22)$ & $8 \times 10^{-5}$ & $8 \times 10^{-5}$ & $8 \times 10^{-5}$ & $8 \times 10^{-5}$ & $8 \times 10^{-5}$ & $8 \times 10^{-5}$ & $8 \times 10^{-5}$ \\
$(12)(23)$ & .08 & $*$ & $1.2 \times 10^{-2}$ & $*$ & $1.2 \times 10^{-2}$ & $1.2 \times 10^{-2}$ & $6 \times 10^{-3}$ \\
$(12)(31)$ & $4 \times 10^{-5}$ & & .1 & & .1 & $4 \times 10^{-5}$ & $4 \times 10^{-5}$ \\
$(12)(32)$ & 1. & 1. & .6 & .4 & .4 & & 1. \\
$(12)(33)$ & .08 & $*$ & .16 & $*$ & .16 & $.16^{-5}$ & .08 \\
\hline
\end{tabular}

Table 2: Generation-mixing leptoquark coupling limits, from ref. [7]. The interactions denoted by $*$ involve a top quark and the corresponding constraints have to be evaluated using the known top quark mass.

combinations

$$
\frac{1}{2} g^{i j} g^{k m}\left(\frac{m_{\mathrm{LQ}}}{100 \mathrm{GeV}}\right)^{2}
$$

In Table 2 we reproduce their results for $g^{1 j} g^{m n}$ at $m_{\mathrm{LQ}}=200 \mathrm{GeV}$.

We see from Table 2 that there are many ways to avoid the bounds and still maintain consistency with the HERA signal. It is still premature to make a detailed study, therefore we mention only one possibility. Suppose that all the couplings are extremely small except (12)(12), i.e. when the leptoquark is coupled to a strange quark and a positron or to a charm quark and a positron. The smaller strange quark and charm quark content of the proton requires larger leptoquark couplings (see Fig. 1) but atomic parity violation constraints are avoided since there is no coupling to a second generation quark in the proton. In this scenario the signal in $e^{-} p$ scattering will be as large as for $e^{+} p$. If we 
insist on a smaller branching ratio $(<1)$ then we may allow either for both left and right handed couplings or for a significant $(12)(23)$ coupling. Another possibility is to allow also for a (12)(31) coupling. In this way the Tevatron mass limit could be relaxed. In this scenario charged current decay modes can also easily be arranged. Finally we note that (i) the (12)(12) solution resembles the squark solution suggested in [11, and (ii) although the above choice may appear somewhat ad hoc we should recall the huge hierarchy of the Yukawa couplings in the Standard Model without scalar leptoquarks.

\section{Leptoquark production at the Tevatron}

Colour-singlet leptoquarks have a standard gauge coupling $\left(g_{s}\right)$ to gluons and can therefore be copiously pair produced in hadron-hadron collisions. The direct coupling to quarks $(\lambda)$ gives additional contributions to the production cross section, but these are numerically much less important for the range of $\lambda$ allowed by the HERA and APV data. In addition, vector leptoquarks can have additional anomalous couplings $\left(\kappa_{V}, \lambda_{V}\right)$ to gluons, analogous to the anomalous electric quadrupole and magnetic dipole moments of $W$ bosons. Although these lead to a violation of unitarity in the production cross section, they may be regarded as an effective low energy parametrization of a more complicated theoretical structure.

Assuming that $\mathrm{LQ} \rightarrow l+q$ is the dominant leptoquark decay mode, the signature of pair production in hadron collisions - two energetic leptons and two energetic jets widely separated in phase space - is rather distinctive. Backgrounds from processes like $W, Z+$ jets, $b \bar{b}+$ jets, etc. can be suppressed in principle by kinematic cuts.

The pair production subprocess cross sections are ${ }^{4}$, with $\beta^{2}=1-4 m_{\mathrm{LQ}}^{2} / \hat{s}$,

$$
\begin{aligned}
\hat{\sigma}_{g g \rightarrow S \bar{S}} & =\frac{\pi \alpha_{S}^{2}}{96 \hat{s}}\left[\beta\left(41-31 \beta^{2}\right)-\left(17-18 \beta^{2}+\beta^{4}\right) \ln \left(\frac{1+\beta}{1-\beta}\right)\right], \\
\hat{\sigma}_{q \bar{q} \rightarrow S \bar{S}} & =\frac{2 \pi \alpha_{S}^{2}}{27 \hat{s}} \beta^{3},
\end{aligned}
$$

for scalar pair production and 13, 14, 15, 16, 17

$$
\begin{aligned}
\hat{\sigma}_{g g \rightarrow V \bar{V}}=\frac{\pi \alpha_{S}^{2}}{96 m_{\mathrm{LQ}}^{2}}[ & \beta\left(\frac{523}{4}-90 \beta^{2}+\frac{93}{4} \beta^{4}\right) \\
& \left.-\frac{3}{4}\left(65-83 \beta^{2}+19 \beta^{4}-\beta^{6}\right) \ln \left(\frac{1+\beta}{1-\beta}\right)\right],
\end{aligned}
$$

\footnotetext{
${ }^{4}$ The cross sections for scalar pair production have been derived in the literature in many different guises. See, for example, ref. [13] and references therein.
} 


$$
\hat{\sigma}_{q \bar{q} \rightarrow V \bar{V}}=\frac{4 \pi \alpha_{S}^{2}}{9 m_{\mathrm{LQ}}^{2}} \frac{\beta^{3}}{24}\left[23-3 \beta^{2}+\frac{4}{1-\beta^{2}}\right],
$$

for vector pair production, with $\lambda_{V}=\kappa_{V}=0$. Note that in general the scattering amplitudes for $q \bar{q} \rightarrow S \bar{S}, V \bar{V}$ receive additional contributions $\left(\hat{\sigma} \sim \alpha_{S} \lambda^{2}, \lambda^{4}\right)$ from diagrams involving direct quark-leptoquark couplings (e.g. $q \bar{q} \rightarrow$ LQ $\overline{\mathrm{LQ}}$ by $t$-channel exchange of a lepton). However for $\lambda^{2}=\mathcal{O}\left(10^{-1}\right)$ or smaller, these contributions are much smaller than the $\mathcal{O}\left(\alpha_{S}^{2}\right)$ contributions listed above. The generalization of (14) including non-zero anomalous couplings is presented in ref. [13].

Fig. 3 shows both cross sections at $\sqrt{s}=1.8 \mathrm{TeV}$ as a function of the leptoquark mass. Note that for $m_{\mathrm{LQ}} \sim 200 \mathrm{GeV}$ the $q \bar{q}$ annihilation subprocesses are dominant. The parton distributions are the $\operatorname{MRS}(\mathrm{R} 2)$ set from ref. [18] (with $\alpha_{S}\left(M_{Z}^{2}\right)=0.12$ ) and the renormalization and factorization scales are set equal to $m_{\mathrm{LQ}}$. We see from Fig. 3 that the cross sections for $m_{\mathrm{LQ}} \sim 200 \mathrm{GeV}$ vector and scalar pair production are $\mathcal{O}(10) \mathrm{pb}$ and $\mathcal{O}(0.1) \mathrm{pb}$ respectively. The former are therefore ruled out by the current Tevatron data [12]. The only caveat to this is that it is possible to 'fine-tune' the anomalous couplings $\kappa_{V}, \lambda_{V}$ to suppress the vector pair cross section by between one and two orders of magnitude, see for example ref. [13. However this would appear to be completely unnatural. The cross sections in Fig. 3 do not include NLO QCD corrections. Estimates for these can be extracted from the calculation of ref. [19] for squark pair production in the infinite gluino mass limit. Numerically, the NLO corrections increase the cross section by a modest $\mathcal{O}(+10 \%)$ in the mass range of interest (see Fig. 19(b) of ref. [19]).

A $200 \mathrm{GeV}$ scalar leptoquark would therefore give rise to approximately 10 events in a sample corresponding to $100 \mathrm{pb}^{-1}$ at the Tevatron. In fact a recent detailed analysis by the D0 collaboration [12] of their combined Run I data gives a $(95 \% \mathrm{cl})$ lower mass limit of $175 \mathrm{GeV}(147 \mathrm{GeV})$ assuming $\mathrm{BR}(\mathrm{LQ} \rightarrow e q)=1(0.5)$. f Thus the $m_{\mathrm{LQ}} \sim 200 \mathrm{GeV}$ (scalar LQ) interpretation of the HERA excess events is allowed, by a small margin. Obviously the Tevatron limit is weakened if the branching ratio to $e q$ is lowered (see section 2 above).

Finally, we show also in Fig. 3 single scalar leptoquark production cross sections from the subprocess $q g \rightarrow \mathrm{LQ}+l[20$ :

$$
\hat{\sigma}_{q g}=\frac{\lambda^{2} \alpha_{S}}{48 \hat{s}}\left[1+6 x-7 x^{2}+4 x(1+x) \ln x\right],
$$

with $x=m_{\mathrm{LQ}}^{2} / \hat{s} \leq 1$. In Fig. 3 we have taken, for illustration, $\lambda^{2}=0.01$ and distinguished the cases where the leptoquark couples to $u, d$ and $s$ quarks. Thus, for example, for a

\footnotetext{
${ }^{5}$ There is as yet no combined Run I published first generation leptoquark mass limit from the CDF collaboration.
} 
scalar leptoquark which is produced at HERA via $e^{+} u$ and has a $100 \%$ branching ratio into the same state, the curve labeled ' $S_{u}$ ' gives the number of $g u \rightarrow\left(e^{+} u\right)+e^{-}$and $g \bar{u} \rightarrow\left(e^{-} \bar{u}\right)+e^{+}$events. Other $\lambda^{2}$ values and branching ratios can be obtained by a simple rescaling of the curves. Note also that for a scalar leptoquark which gives rise also to charged current events are HERA, for example via $e^{+} d \rightarrow S \rightarrow \bar{\nu}_{e} u$, the additional 'missing energy' processes $g d \rightarrow\left(\bar{\nu}_{e} u\right)+e^{-}$and $g u \rightarrow\left(\bar{\nu}_{e} u\right)+\nu_{e}$ are possible. The corresponding event rates are readily estimated from the curves in Fig. 3 .

The most important conclusion from the single leptoquark production curves in Fig. 3 is that for $m_{\mathrm{LQ}} \sim 200 \mathrm{GeV}$, the single production cross section is less than the pair production cross section for $\lambda^{2}<\mathcal{O}(0.07,0.16,0.8)$ for coupling to $u, d, s$ quarks.

\section{Production cross section in NLO at HERA}

In order to get an accurate value of the leptoquark coupling constant from the HERA data one should take into account the next-to-leading order QCD corrections. This is also required for consistency if parton distributions obtained with a NLO fitting procedure are used. In this section we describe the calculation for scalar leptoquarks since, as we have seen, vector particles are disfavoured by searches at the Tevatron. Note that the transverse momentum $\left(k_{T}\right)$ distribution of the leptoquarks with respect to the beam direction is a by-product of the full NLO correction.

The NLO-corrected cross section can be written as

$$
\begin{aligned}
& \sigma\left(e^{+} q\right)=\frac{\pi \lambda^{2}}{4 s} \int_{x}^{1} \frac{d z}{z} {\left[q\left(\frac{x}{z}, \mu_{I R}^{2}\right)\left\{\delta(1-z)+\frac{\alpha_{S}\left(\mu^{2}\right)}{2 \pi} C_{F} K_{q}(z)\right\}\right.} \\
&\left.+g\left(\frac{x}{z}, \mu_{I R}^{2}\right) \frac{\alpha_{S}\left(\mu^{2}\right)}{2 \pi} T_{R} K_{g}(z)\right] .
\end{aligned}
$$

where $x=m_{\mathrm{LQ}}^{2} / s$ and $C_{F}=4 / 3, T_{R}=1 / 2$. The coefficient functions are calculated from the $\mathcal{O}\left(\alpha_{S}\right)$ Feynman diagrams. Using $\overline{\mathrm{MS}}$ factorization and renormalization we obtain (see Appendix for more details)

$$
\begin{aligned}
K_{q}(z)= & \delta(1-z)\left(-\frac{\pi^{2}}{3}+\frac{3}{2} \ln \frac{\mu_{U V}^{2}}{\mu_{I R}^{2}}\right)-\frac{2 z}{(1-z)_{+}}, \\
& +2\left(1+z^{2}\right)\left(\frac{\ln (1-z)}{1-z}\right)_{+}-\ln \left(\frac{z \mu_{I R}^{2}}{m^{2}}\right) \frac{1+z^{2}}{(1-z)_{+}} \\
K_{g}(z)= & {\left[(1-z)^{2}+z^{2}\right] \ln \left(\frac{z(1-z)^{2} \mu_{I R}^{2}}{m^{2}}\right)+2 z(1-z)(2+\ln z) . }
\end{aligned}
$$


Here $\mu_{I R}^{2}$ and $\mu_{U V}^{2}$ denote the factorization and (ultraviolet) renormalization[ scales respectively. In our numerical calculations we will use

$$
\mu^{2}=\mu_{I R}^{2}=\mu_{U V}^{2}=m_{\mathrm{LQ}}^{2} .
$$

Note that the QCD correction is universal for the different types of scalar leptoquarks discussed in section 1 .

Figure 1 shows the NLO-corrected production cross sections for $e^{+} q \rightarrow \mathrm{LQ}$ with $q=u, d, \bar{u}, \bar{d}, s$ at $\sqrt{s}=300 \mathrm{GeV}$. For ease of comparison we have set the overall leptoquark coupling to unity, i.e. $\lambda^{2}=1$. The parton distributions are the MRS(R2) set from ref. [18], with $\alpha_{S}\left(M_{Z}^{2}\right)=0.120$. The cross section hierarchy mainly reflects the hierarchy of the quark distributions at large $x$. The ratio of the NLO to LO contributions (the ' $\mathrm{K}$-factor' $\mathcal{K})$ is non-negligible. For the valence quark cross sections, $\mathcal{K}$ increases from about 1.25 to 1.45 in the mass range of interest, $175 \mathrm{GeV}<m_{\mathrm{LQ}}<225 \mathrm{GeV}$, while for the sea quarks $\mathcal{K}$ increases from about 1.3 to 1.6 in the same mass range. Table 6 lists cross section values for leptoquark masses relevant to the HERA high $Q^{2}$ events. In the limit $m_{\mathrm{LQ}} \rightarrow \sqrt{s}$, i.e. $x \rightarrow 1$, the correction is dominated by a soft-gluon double logarithm, $\mathcal{K} \sim 1+\alpha_{S} C_{F} \ln ^{2}(1-x) / \pi$.

\begin{tabular}{|c|c|c|c|c|c|}
\hline & $\sigma\left(e^{+} u\right)$ & $\sigma\left(e^{+} d\right)$ & $\sigma\left(e^{+} \bar{u}\right)$ & $\sigma\left(e^{+} \bar{d}\right)$ & $\sigma\left(e^{+} s\right)$ \\
\hline$m_{\mathrm{LQ}}=175 \mathrm{GeV}$ & 3289 & 1026 & 47.0 & 99.6 & 49.7 \\
& $(2659)$ & $(801)$ & $(35.7)$ & $(73.8)$ & $(37.9)$ \\
\hline$m_{\mathrm{LQ}}=200 \mathrm{GeV}$ & 1436 & 352 & 7.32 & 16.7 & 8.21 \\
& $(1096)$ & $(260)$ & $(5.20)$ & $(11.5)$ & $(5.82)$ \\
\hline$m_{\mathrm{LQ}}=225 \mathrm{GeV}$ & 475 & 89.1 & 0.634 & 1.52 & 0.740 \\
& $(337)$ & $(61.3)$ & $(0.413)$ & $(0.96)$ & $(0.479)$ \\
\hline
\end{tabular}

Table 3: Leptoquark cross sections (in pb) at NLO in $e^{+} p$ collisions at $\sqrt{s}=300 \mathrm{GeV}$, assuming unit coupling $\lambda^{2}=1$. The numbers in brackets are the corresponding leadingorder cross sections.

The transverse momentum distribution $d \sigma / d k_{T}^{2}$ and the average transverse momentum squared $\left\langle k_{T}^{2}\right\rangle$ of the leptoquarks can also be calculated from the $O\left(\alpha_{S}\right)$ diagrams for the

\footnotetext{
${ }^{6}$ The leptoquark coupling $\lambda^{2}$ is renormalized by the $\mathcal{O}\left(\alpha_{S}\right)$ vertex corrections, see Appendix, hence $\lambda^{2} \equiv \lambda^{2}\left(\mu_{U V}^{2}\right)$.
} 
processes $e q \rightarrow g \mathrm{LQ}$ and $e g \rightarrow q \mathrm{LQ}$ (see Appendix A.4). For the average we obtain

$$
\frac{\left\langle k_{T}^{2}\right\rangle}{m_{\mathrm{LQ}}^{2}}=\frac{\pi \lambda^{2}}{4 s \sigma} \frac{\alpha_{S}\left(\mu^{2}\right)}{2 \pi} \int_{x}^{1} \frac{d z}{z^{2}}\left[q\left(\frac{x}{z}, \mu_{I R}^{2}\right) C_{F} \bar{K}_{q}(z)+g\left(\frac{x}{z}, \mu_{I R}^{2}\right) T_{R} \bar{K}_{g}(z)\right],
$$

where $\sigma$ is defined in eq. (16) and

$$
\begin{aligned}
& \bar{K}_{q}(z)=\frac{1}{3}\left(1+z^{2}\right)(1-z) \\
& \bar{K}_{g}(z)=\frac{1}{2}-3 z+\frac{z^{2}}{2}+2 z^{3}-4 z^{2} \ln z
\end{aligned}
$$

Figure 2 shows $\sqrt{\left\langle k_{T}^{2}\right\rangle}$ as a function of $m_{\mathrm{LQ}}$ for the various $e^{+} q$ production mechanisms. Note that the average transverse momentum is in general very small, because the emission of an additional energetic parton is heavily suppressed.

\section{Conclusions}

We have calculated the NLO QCD corrections to the production cross-section of a coloured s-channel scalar resonance (leptoquark or squark) at HERA and reevaluated various leptoquark production cross sections for Tevatron. The average transverse momentum of the leptoquark produced at HERA was also calculated from the NLO result. Our calculation was motivated by the leptoquark interpretation of the excess of events found by $\mathrm{H} 1$ and ZEUS at HERA in $e^{+} p$ deep-inelastic scattering above the Standard Model prediction. The simplest and perhaps most attractive 'new physics' interpretation of the data is in terms of a first family leptoquark with a single Yukawa coupling. Constraints from data on atomic parity violation, double beta decay and various flavour changing processes are consistent with the HERA data only for isodoublet scalar leptoquark production. This interpretation, however, is seriously challenged by severe constraints from leptoquark pair production at the Tevatron $p \bar{p}$ collider which is largely independent from the Yukawa coupling of the leptoquark. The Tevatron limits are even more severe for vector leptoquarks. Because the QCD corrections to the HERA cross sections are large and positive, the extracted couplings are somewhat smaller than those obtained from a leading order analysis only.

If one also considers leptoquarks which couple to several generations, then in the enlarged space of couplings one can find several solutions compatible with the low energy constraints and the HERA excess events. In particular, one can have solutions with reduced branching ratios and/or charged current events, as well as lepton number violating 
processes. Furthermore one can no longer rule out the isosinglet and isotriplet scalar leptoquark interpretation in such models. The solutions with reduced branching ratios also relax the Tevatron limits. However, they do require a rather ad hoc hierarchy in the Yukawa couplings (although such a strong hierarchy already exists in the Standard Model Higgs couplings).

The strong hierarchy in the Yukawa couplings in the allowed solutions implies a very specific flavour structure and therefore such models can be tested when more HERA data are available. We have considered, as a specific example, the case where the leptoquark is dominantly coupled to a positron and a strange squark.

Note added: Since the H1 and ZEUS results were made public, there have been many theoretical papers attempting to explain the apparent event excess in terms of leptoquarktype particles [2]. The present study overlaps with some of these in some respects, but to our knowledge our calculation of the HERA cross sections is the first to include the $\mathcal{O}\left(\alpha_{S}\right)$ QCD corrections. 


\section{Appendix}

\section{A.1 Kinematics and leading-order cross section}

We consider the process

$$
\begin{gathered}
e^{+}\left(p_{2}\right)+q\left(p_{1}\right) \rightarrow R(q) \\
p_{1}+p_{2}=q, \quad \hat{s}=\left(p_{1}+p_{2}\right)^{2}=q^{2}=m^{2}, \quad p_{1}^{2}=p_{2}^{2}=0 .
\end{gathered}
$$

The leading order transition amplitude is

$$
T_{\mathrm{B}}=\lambda \bar{v}\left(p_{2}\right) P_{\mathrm{L}, \mathrm{R}} u\left(p_{1}\right), \quad P_{\mathrm{L}, \mathrm{R}}=\frac{1 \pm \gamma_{5}}{2} .
$$

The spin- and colour-averaged squared amplitude is

$$
{\overline{\mid T_{\mathrm{B}}}}^{2}=\frac{\lambda^{2} \hat{s}}{4}
$$

which gives the leading-order cross sections

$$
\begin{gathered}
\hat{\sigma}_{0}(\hat{s})=\frac{\pi \lambda^{2}}{4} \delta\left(\hat{s}-m^{2}\right) \\
\sigma_{\mathrm{ep}}=\int d y q(y) \hat{\sigma}_{0}(\hat{s}=y s)=\frac{\pi \lambda^{2}}{4 s} q(x), \quad \text { with } m^{2}=x s .
\end{gathered}
$$

\section{A.2 Virtual corrections}

In the calculation of the real and virtual corrections, we regulate the infra-red and ultraviolet divergences using dimensional regularization with $d=4-2 \epsilon$.

\section{A.2.1 Loop integrals}

We need the loop integrals over two propagators:

$$
\begin{gathered}
I_{2}\left(q^{2}\right)=\int d^{d} k \frac{1}{\left(k^{2}+i \eta\right)\left[(k-q)^{2}+i \eta\right]}=Q_{-q^{2}} T_{0}^{\mathrm{UV}}, \quad q^{2} \neq 0, \\
Q_{-q^{2}}=i \pi^{d / 2} \Gamma(1+\epsilon)\left(-q^{2}-i \eta\right)^{-\epsilon}
\end{gathered}
$$




$$
\begin{aligned}
& T_{0}^{\mathrm{UV}, \mathrm{IR}}=\frac{1}{\epsilon_{\mathrm{UV}, \mathrm{IR}}}+2, \\
I_{2}\left(q^{2}\right)= & i \pi^{2} \frac{(4 \pi)^{\epsilon}}{\Gamma(1-\epsilon)}\left(T_{0}^{\mathrm{UV}}-T_{0}^{\mathrm{IR}}=0 \text { if } q^{2}=0,\right. \\
I_{2}(q)_{\mu}= & \int d^{d} k \frac{k_{\mu}}{\left(k^{2}+i \eta\right)\left[(k-q)^{2}+i \eta\right]}=\frac{1}{2} q_{\mu} I\left(q^{2}\right), \\
I_{2}\left(q^{2} ; m^{2}\right)= & \int d^{d} k \frac{1}{\left(k^{2}+i \eta\right)\left[(k-q)^{2}-m^{2}+i \eta\right]} \\
I_{2}\left(m^{2} ; m^{2}\right)= & Q_{m^{2}} \frac{1}{\epsilon} \int_{0}^{1} d x\left[x-x(1-x) \frac{q^{2}}{m^{2}}\right]^{-\epsilon}, \\
I_{2}^{\prime}\left(m^{2} ; m^{2}\right)= & \left.m^{2} \frac{d I_{2}\left(q^{2} ; m^{2}\right)}{d q^{2}}\right|_{q^{2}=m^{2}}=-Q_{m^{2}} \frac{1}{2} T_{0}^{\mathrm{IR}},
\end{aligned}
$$

and the loop integral over three propagators:

$$
\begin{aligned}
I_{3}(q, p)= & \int d^{d} k \frac{1}{\left(k^{2}+i \eta\right)\left[(k-q)^{2}+i \eta\right]\left[(k-p)^{2}+i \eta\right]}, \\
& q^{2} \neq 0, p^{2}=0,(q-p)^{2}=0 \\
I_{3}(q, p)= & -Q_{-q^{2}}\left(-\frac{1}{q^{2}}\right) R_{0}, \quad R_{0}=\frac{1}{\epsilon^{2}}-\frac{\pi^{2}}{6}+\mathcal{O}(\epsilon), \\
I_{3}\left(q^{2} ; m^{2}\right)= & \int d^{d} k \frac{1}{\left(k^{2}+i \eta\right)\left[(k-q)^{2}-m^{2}+i \eta\right]\left[(k-p)^{2}+i \eta\right]}, \\
I_{3}\left(m^{2} ; m^{2}\right)= & \frac{1}{m^{2}} Q_{m^{2}}\left(-\frac{1}{2 \epsilon^{2}}\right) .
\end{aligned}
$$

It is interesting to compare the massless and massive $I_{3}$ integrals: the double pole singularities have opposite sign and different normalization, and the finite terms are also different. We note also the relation

$$
\Gamma(1+\epsilon)=\frac{1}{\Gamma(1-\epsilon)}\left(1+\epsilon^{2} \frac{\pi^{2}}{6}\right)+\mathcal{O}\left(\epsilon^{3}\right)
$$

\section{A.2.2 Self-energy corrections}

Self-energy corrections give wave-function renormalization through the relations

$$
T_{\mathrm{S}}=\left.\frac{1}{2} T_{\mathrm{B}} \frac{d \Sigma_{\mathrm{S}}}{d q^{2}}\right|_{q^{2}=m^{2}}, \quad T_{\mathrm{F}}=\left.\frac{1}{2} T_{\mathrm{B}} \frac{d \Sigma_{\mathrm{F}}}{d \not p}\right|_{p^{2}=0}
$$


where $T_{\mathrm{B}}$ denotes the Born amplitude (see eq. (A3)) and $-i \Sigma_{\mathrm{S}},-i \Sigma_{\mathrm{F}}$ are given by the selfenergy diagrams for massive scalar and massless fermion lines respectively. Introducing the auxiliary notation

$$
D_{1}=(k-q)^{2}-m^{2}, \quad D_{2}=\left(k-p_{1}\right)^{2}, \quad D_{3}=k^{2}, \quad C=-i C_{F}(2 \pi)^{-d} g_{s}^{2} \mu^{2 \epsilon},
$$

where $C_{F}=4 / 3$ is the usual colour factor, we can write

$$
\begin{aligned}
\Sigma_{\mathrm{S}} & =C \int d^{d} k \frac{(2 q-k)^{2}}{D_{1} D_{3}}=C \int d^{d} k \frac{2 D_{1}-D_{3}+2\left(q^{2}+m^{2}\right)}{D_{1} D_{3}} \\
& =-C \int d^{d} k \frac{1}{k^{2}-m^{2}}+2 C\left(q^{2}+m^{2}\right) I_{2}\left(q^{2}, m^{2}\right) .
\end{aligned}
$$

Therefore

$$
\left.\frac{d \Sigma_{\mathrm{S}}}{d q^{2}}\right|_{q^{2}=m^{2}}=2 C\left[I_{2}\left(m^{2} ; m^{2}\right)+2 I_{2}^{\prime}\left(m^{2} ; m^{2}\right)\right]
$$

and

$$
\left.\frac{1}{2} \frac{d \Sigma_{\mathrm{S}}}{d q^{2}}\right|_{q^{2}=m^{2}}=C Q_{m^{2}}\left(T_{0}^{\mathrm{UV}}-T_{0}^{\mathrm{IR}}\right)=0
$$

where

$$
C Q_{m^{2}}=C_{F} \frac{\alpha_{S}}{4 \pi}\left(\frac{4 \pi \mu^{2}}{m^{2}}\right)^{\epsilon} \Gamma(1+\epsilon)
$$

The self-energy of the massive scalar is zero. As in the case of the massless fermion correction, the ultraviolet and infra-red divergences exactly cancel. The ultraviolet counter term is non-zero

$$
\left(\frac{1}{2} \Sigma_{\mathrm{S}}^{\prime}\right)^{\mathrm{CT}}=C_{F} \frac{\alpha_{S}}{4 \pi}\left(\frac{4 \pi \mu^{2}}{m^{2}}\right)^{\epsilon} \Gamma(1+\epsilon)\left(-\frac{1}{\epsilon}+\ln \frac{\mu^{2}}{m^{2}}\right) .
$$

Similarly for the massless fermion line we obtain

$$
\begin{aligned}
\Sigma_{\mathrm{F}} & =C(2-d) \int d^{d} k \frac{\not_{1}-\not k}{D_{2} D_{3}}=-2 C(d / 2-1) \frac{1}{2} \not_{1} I_{2}\left(p_{1}^{2} ; 0\right) \\
& =C_{F} \frac{\alpha_{S}}{4 \pi} \frac{(4 \pi)^{\epsilon}}{\Gamma(1-\epsilon)}\left(-T_{0}^{\mathrm{UV}}+T_{0}^{\mathrm{IR}}\right)
\end{aligned}
$$

and therefore

$$
\left.\frac{1}{2} \frac{d \Sigma_{\mathrm{F}}}{d p_{1}}\right|_{\mathbb{P}_{1}=0}=C_{F} \frac{\alpha_{S}}{4 \pi} \frac{(4 \pi)^{\epsilon}}{\Gamma(1-\epsilon)}\left(-\frac{1}{2} T_{0}^{\mathrm{UV}}+\frac{1}{2} T_{0}^{\mathrm{IR}}\right)=0 .
$$

In dimensional regularization, the self-energy of the massless fermion is zero: the ultraviolet and infra-red divergences exactly cancel. Using the approximate relation (A19) the ultraviolet counter term can be written as

$$
\left(\frac{1}{2} \Sigma_{\mathrm{F}}^{\prime}\right)^{\mathrm{CT}}=C_{F} \frac{\alpha_{S}}{4 \pi}\left(\frac{4 \pi \mu^{2}}{m^{2}}\right)^{\epsilon} \Gamma(1+\epsilon)\left(\frac{1}{2 \epsilon}-\frac{1}{2} \ln \frac{\mu^{2}}{m^{2}}\right) .
$$




\section{A.2.3 Vertex corrections}

The numerator of the vertex correction is given by

$$
\begin{aligned}
\bar{u}\left(p_{2}\right)\left(-\not k+\not p_{1}\right)\left(-\not k+2 \not p_{2}\right) u\left(p_{1}\right) & =\bar{u}\left(p_{2}\right)\left[k^{2}-2 p_{1} k-4 p_{2} k+4 p_{1} p_{2}\right] u\left(p_{1}\right) \\
& =T_{\mathrm{B}}\left(k^{2}-4 k q+2 p_{1} k+2 m^{2}\right) \\
& =T_{\mathrm{B}}\left(2 D_{1}-D_{2}+2 m^{2}\right) .
\end{aligned}
$$

The vertex correction can then be written as

$$
\begin{aligned}
V & =T_{\mathrm{B}} C \int d^{d} k \frac{2 D_{1}-D_{2}+2 m^{2}}{D_{1} D_{2} D_{3}} \\
& =2 m^{2} C I_{3}\left(0, m^{2} ; m^{2}\right)+2 C I_{2}\left(p_{2}^{2}\right)-C I_{2}\left(m^{2} ; m^{2}\right) \\
& =C Q_{m^{2}}\left(-\frac{1}{\epsilon^{2}}-\frac{1}{\epsilon}-2+\mathcal{O}(\epsilon)\right)
\end{aligned}
$$

Ultraviolet divergences appear in $I_{2}\left(p_{2}^{2}\right)$ and $I_{2}\left(m^{2}, m^{2}\right)$, and therefore the counter term is

$$
V^{\mathrm{CT}}=T_{\mathrm{B}} C_{F} \frac{\alpha_{S}}{4 \pi}\left(\frac{4 \pi \mu^{2}}{m^{2}}\right)^{\epsilon} \Gamma(1+\epsilon)\left(-\frac{1}{\epsilon}+\ln \frac{\mu^{2}}{m^{2}}\right) .
$$

\section{A.2.4 Contribution to the subprocess cross section}

The total contribution of the virtual corrections to the subprocess cross section is

$$
\begin{aligned}
\hat{\sigma}^{\text {virt }} & =2 \hat{\sigma}_{0}\left[\frac{1}{2} \Sigma_{\mathrm{S}}^{\prime}+\frac{1}{2} \Sigma_{\mathrm{F}}^{\prime}+V / T_{\mathrm{B}}+\left(\frac{1}{2} \Sigma_{\mathrm{S}}^{\prime}+\frac{1}{2} \Sigma_{\mathrm{F}}^{\prime}+V / T_{\mathrm{B}}\right)^{\mathrm{CT}}\right] \\
& =\hat{\sigma}_{0} C_{F} \frac{\alpha_{S}}{2 \pi}\left(\frac{4 \pi \mu^{2}}{m^{2}}\right)^{\epsilon} \Gamma(1+\epsilon)\left(-\frac{1}{\epsilon^{2}}-\frac{5}{2 \epsilon}-2+\frac{3}{2} \ln \frac{\mu_{\mathrm{UV}}^{2}}{m^{2}}\right),
\end{aligned}
$$

which leads to the final result

$$
\sigma^{\mathrm{virt}}=C_{F} \frac{\lambda^{2} \pi}{4 \hat{s}} \frac{\alpha_{S}}{2 \pi}\left(\frac{4 \pi \mu^{2}}{m^{2}}\right)^{\epsilon} \frac{1}{\Gamma(1-\epsilon)} K_{q}^{(\mathrm{v})},
$$

where

$$
K_{q}^{(\mathrm{v})}=\delta(1-z)\left(-\frac{1}{\epsilon^{2}}-\frac{5}{2 \epsilon}-2-\frac{\pi^{2}}{6}+\frac{3}{2} \ln \frac{\mu_{\mathrm{UV}}^{2}}{m^{2}}+\mathcal{O}(\epsilon)\right)
$$

\section{A.3 Real contributions}




\section{A.3.1 Kinematics, matrix elements, counter terms}

We consider the processes

$$
\begin{gathered}
e^{+}\left(p_{2}\right)+q\left(p_{1}\right) \rightarrow g(k)+\mathrm{LQ}(q), \\
e^{+}\left(p_{2}\right)+g\left(p_{1}\right) \rightarrow q(k)+\mathrm{LQ}(q)
\end{gathered}
$$

The kinematical variables are

$$
q^{2}=m^{2}, \quad \hat{s}=2 p_{1} p_{2}=m^{2}+2 k q, \quad \hat{t}=-2 k p_{1}, \quad \hat{u}=-2 k p_{2} .
$$

The spin- and colour-averaged matrix element squared of process (A36) is given by

$$
{\overline{\mid T_{q}}}^{2}=\frac{\lambda^{2}}{4} C_{F} g_{s}^{2} \psi\left(p_{2}, p_{1} ; k, q\right)_{q} \quad \text { and } \quad \hat{\sigma}^{(0)}(\hat{s})_{q}=\frac{\lambda^{2}}{4} C_{F} g_{s}^{2} R\left[\psi\left(p_{2}, p_{1} ; k, q\right)_{q}\right]
$$

where $R[]$ denotes the phase-space integral times the flux factor $\mu^{2 \epsilon} /(2 \hat{s})$. From explicit calculation of the two Feynman diagrams we obtain

$$
\begin{aligned}
\psi\left(p_{2}, p_{1} ; k, q\right)_{q}= & \frac{m^{4}}{(k q)\left(k p_{1}\right)}-\frac{m^{4}}{(k q)^{2}}+\frac{2 m^{2}}{k p_{1}}+\frac{2 k q}{k p_{1}} \\
& -\frac{2 m^{2}}{k q}-2+2 \epsilon-2 \epsilon \frac{k q}{k p_{1}}
\end{aligned}
$$

Similarly, for the process (A37) we obtain

$$
{\overline{\left|T_{g}\right|}}^{2}=\frac{\lambda^{2}}{4} C_{F} g_{s}^{2} \psi\left(p_{2}, p_{1} ; k, q\right)_{g} .
$$

where due to crossing symmetry

$$
\psi_{2}\left(p_{2}, p_{1} ; k, q\right)_{g}=-\frac{1}{1-\epsilon} \psi_{2}\left(p_{2},-k ;-p_{1}, q\right)_{q} .
$$

Note that here we take into account the fact that the spin average factor for an initial gluon is $2(1-\epsilon)$. The subprocess cross section is obtained by adding the $\overline{\mathrm{MS}}$ counter term

$$
\sigma_{a}^{\mathrm{CT}}=\frac{\alpha_{S}}{2 \pi}(4 \pi)^{\epsilon} \frac{1}{\epsilon \Gamma(1-\epsilon)} \int_{0}^{1} d z P_{b / a}(z, 0) \hat{\sigma}_{b}^{\mathrm{B}}(z \hat{s}),
$$

where $P_{b / a}(z, \epsilon)$ denotes the $\epsilon$ dependent and spin-independent Altarelli-Parisi splitting functions. In the present context we need

$$
\begin{aligned}
P_{q / q}(z, \epsilon) & =C_{F}\left[\frac{1+z^{2}}{(1-z)_{+}}-\epsilon(1-z)+\frac{3}{2} \delta(1-z)\right], \\
P_{q / g}(z, \epsilon) & =\frac{T_{R}}{1-\epsilon}\left[z^{2}+(1-z)^{2}-\epsilon\right] .
\end{aligned}
$$


To perform the phase-space integrals it is convenient to introduce energy-angle variables in the subprocess centre-of-mass frame:

$$
\begin{aligned}
p_{1}^{\mu} & =\frac{\sqrt{\hat{s}}}{2}(1,0,0,1), \quad p_{2}^{\mu}=\frac{\sqrt{\hat{s}}}{2}(1,0,0,-1) \\
k & =\frac{\sqrt{\hat{s}}}{2}(1-z)(1,0, \sin \theta, \cos \theta), \quad q=p_{1}+p_{2}-k .
\end{aligned}
$$

Changing variables to

$$
z=m^{2} / \hat{s} \quad \text { and } \quad u=\frac{1}{2}(1-\cos \theta),
$$

gives, for the $d$-dimensional phase space integral times flux factor,

$$
R[]=\frac{1}{32 \pi \hat{s}}\left(\frac{4 \pi \mu^{2}}{\hat{s}^{2}}\right)^{\epsilon} \frac{1}{\Gamma(1-\epsilon)} \int_{0}^{1} d u 2 u^{-\epsilon}(1-u)^{-\epsilon}(1-z)^{1-2 \epsilon} .
$$

In terms of the variables $z$ and $u$ the Lorentz invariant scalar products are

$$
m^{2}=z \hat{s}, \quad k p_{1}=\frac{\hat{s}}{2} u(1-z), \quad k q=\frac{s}{2}(1-z) \quad \text { and } \quad k_{T}^{2}=\hat{s}(1-z)^{2} u(1-u) .
$$

\section{A.3.2 Contribution to $\hat{\sigma}_{1}$ from the $e^{+} q \rightarrow g$ LQ process}

In terms of the cms variables, the $\psi_{q}$ function (see eq. (A40) becomes

$$
\psi\left(p_{1}, p_{2} ; k, q\right)_{q}=4\left\{\frac{1}{2 u(1-z)}\left[\frac{1+z^{2}}{1-z}-\epsilon(1-z)\right]-\frac{z}{(1-z)^{2}}-\frac{1}{2}+\frac{1}{2} \epsilon\right\}
$$

Introducing notations for the integrals

$$
\begin{aligned}
I_{-1} & =\int_{0}^{1} d u u^{-1-\epsilon}(1-u)^{-\epsilon}=-\frac{1}{\epsilon}+\epsilon \frac{\pi^{2}}{6}+\ldots \\
I_{0} & =\int_{0}^{1} d u u^{-\epsilon}(1-u)^{-\epsilon}=1+2 \epsilon+\ldots
\end{aligned}
$$

and for the functions of $z$

$$
\begin{aligned}
V_{-1} & =(1-z)^{-1-2 \epsilon}=-\frac{1}{2 \epsilon} \delta(1-z)+\frac{1}{(1-z)_{+}}-2 \epsilon\left(\frac{\ln (1-z)}{1-z}\right)_{+} \\
V_{0} & =1, \quad V_{1}=(1-z)
\end{aligned}
$$

then

$$
\begin{aligned}
R\left[\psi_{q}\right] & =\frac{1}{8 \pi \hat{s}}\left(\frac{4 \pi \hat{s}^{2}}{m^{2}}\right)^{\epsilon} \frac{1}{\Gamma(1-\epsilon)}\left[I_{-1} V_{-1}\left(1+z^{2}\right)\right. \\
& \left.-\epsilon I_{-1} V_{1}-2 z V_{-1} I_{0}-I_{0} V_{1}\right]
\end{aligned}
$$


and the unsubtracted cross section becomes

$$
\hat{\sigma}_{1 q}=C_{F} \frac{\lambda^{2} \pi}{4 \hat{s}} \frac{\alpha_{S}}{2 \pi}\left(\frac{4 \pi \mu^{2}}{\hat{s}^{2}}\right)^{\epsilon} \frac{1}{\Gamma(1-\epsilon)} \tilde{K}_{q}
$$

where

$$
\begin{aligned}
\tilde{K}_{q}= & \delta(1-z)\left[\frac{1}{\epsilon^{2}}+\frac{1}{\epsilon}+2-\frac{\pi^{2}}{6}\right] \\
& -\frac{1}{\epsilon} \frac{1+z^{2}}{(1-z)_{+}}-\frac{2 z}{(1-z)_{+}}+2\left(\frac{\ln (1-z)}{1-z}\right)_{+}\left(1+z^{2}\right) .
\end{aligned}
$$

The $\overline{\mathrm{MS}}$ counter term cross section (see eqs. (A5, A43)) for this process is

$$
\begin{aligned}
\sigma_{1 q}^{\mathrm{CT}}= & C_{F} \frac{\lambda^{2} \pi}{4 \hat{s}} \frac{\alpha_{S}}{2 \pi}\left(\frac{4 \pi \mu^{2}}{\hat{s}}\right)^{\epsilon} \frac{1}{\Gamma(1-\epsilon)}\left[\frac{1}{\epsilon} \frac{1+z^{2}}{(1-z)_{+}}\right. \\
& \left.+\frac{3}{2} \delta(1-z)-\ln \frac{\mu^{2}}{\hat{s}} \frac{1}{C_{F}} P_{q / q}(z, 0) .\right]
\end{aligned}
$$

Finally for the subtracted partonic cross section we get

$$
\hat{\sigma}_{1 q}^{\mathrm{sub}}=C_{F} \frac{\lambda^{2} \pi}{4 \hat{s}} \frac{\alpha_{S}}{2 \pi}\left(\frac{4 \pi \mu^{2}}{m^{2}}\right)^{\epsilon} \frac{1}{\Gamma(1-\epsilon)} K_{q}^{(\mathrm{r})}
$$

where

$$
\begin{aligned}
K_{q}^{(\mathrm{r})}= & \delta(1-z)\left[\frac{1}{\epsilon^{2}}+\frac{5}{2 \epsilon}+2-\frac{\pi^{2}}{6}-\frac{3}{2} \ln \frac{\mu_{\mathrm{IR}}^{2}}{\hat{s}}\right] \\
& -\frac{2 z}{(1-z)_{+}}+2\left(\frac{\ln (1-z)}{1-z}\right)_{+}\left(1+z^{2}\right)-\ln \frac{\mu_{\mathrm{IR}}^{2}}{\hat{s}} \frac{1+z^{2}}{(1-z)_{+}}
\end{aligned}
$$

\section{A.3.3 Contribution to $\hat{\sigma}_{1}$ from the $e^{+} g \rightarrow q$ LQ process}

In terms of the cms variables, the $\psi_{g}$ function (see eqs. (A40, A42)) becomes

$$
\psi\left(p_{2}, p_{1} ; k, q\right)=\frac{4}{1-\epsilon}\left[\frac{1}{2 u(1-z)} \psi_{1}(z, u)+\psi_{2}(z, u)\right]
$$

where

$$
\begin{aligned}
\psi_{1}(z, u) & =1-2 z+\frac{2 z^{2}}{1-u(1-z)}-\epsilon-(1-\epsilon) u(1-z) \\
\psi_{1}(z, 0) & =z^{2}+(1-z)^{2}-\epsilon \\
\psi_{2}(z, u) & =\frac{1}{2}+\frac{z^{2}}{[1-u(1-z)]^{2}}-\frac{z}{1-u(1-z)}
\end{aligned}
$$


The phase-space integral over $\psi_{g}$ including the flux factor can be written as

$$
R\left[\psi_{g}\right]=\frac{1}{8 \pi \hat{s}}\left(\frac{4 \pi \mu^{2}}{\hat{s}^{2}}\right)^{\epsilon} \frac{1}{\Gamma(1-\epsilon)} \tilde{K}_{g}
$$

where

$$
\begin{aligned}
\tilde{K}_{g}= & \left\{\int_{0}^{1} d u\left[-\frac{1}{\epsilon} \delta(u)+\frac{1}{u_{+}}\right](1+\epsilon)[1-2 \epsilon \ln (1-z)] \psi_{1}(z, u)\right. \\
& \left.+2(1-z) \int_{0}^{1} d u \psi_{2}(z, u)\right\}
\end{aligned}
$$

which leads to

$$
\begin{aligned}
\tilde{K}_{g}= & \left\{-\frac{1}{\epsilon}\left[z^{2}+(1-z)^{2}\right]+2 \ln (1-z)\left[z^{2}+(1-z)^{2}\right]\right. \\
& \left.+4 z-4 z^{2}+2 z \ln z-2 z^{2} \ln ^{2} z\right\} .
\end{aligned}
$$

In this case the counter term simply cancels the $1 / \epsilon$ term and introduces the usual $\ln \mu^{2}$ factor of the $\overline{M S}$ prescription. Finally we get

$$
\hat{\sigma}_{1 g}^{\text {sub }}=T_{R} \frac{\lambda^{2} \pi}{4 \hat{s}} \frac{\alpha_{S}}{2 \pi}\left(\frac{4 \pi \mu^{2}}{m^{2}}\right)^{\epsilon} \frac{1}{\Gamma(1-\epsilon)} K_{g},
$$

where

$$
K_{g}=-\ln \left(\frac{\mu_{I R}^{2}}{\hat{s}(1-z)^{2}}\right)\left[z^{2}+(1-z)^{2}\right]+2 z(1-z)(2+\ln z)
$$

\section{A.3.4 Finite subprocess cross sections}

The finite subprocess cross sections are obtained by adding the virtual and real contributions, whereupon the remaining soft and collinear singularities cancel:

$$
\begin{aligned}
& \hat{\sigma}_{1 q}=\frac{\lambda^{2} \pi}{4 \hat{s}} \frac{\alpha_{S}}{2 \pi} C_{F} K_{q} \\
& \hat{\sigma}_{1 g}=\frac{\lambda^{2} \pi}{4 \hat{s}} \frac{\alpha_{S}}{2 \pi} T_{R} K_{g}
\end{aligned}
$$

where

$$
\begin{aligned}
K_{q}= & K_{q}^{(\mathrm{v})}+K_{q}^{(\mathrm{r})} \\
= & \delta(1-z)\left(-\frac{\pi^{2}}{3}+\frac{3}{2} \ln \frac{\mu_{U V}^{2}}{\mu_{I R}^{2}}\right) \\
& -\frac{2 z}{(1-z)_{+}}+2\left(\frac{\ln (1-z)}{1-z}\right)_{+}\left(1+z^{2}\right)-\ln \frac{\mu_{\mathrm{IR}}^{2}}{\hat{s}} \frac{1+z^{2}}{(1-z)_{+}},
\end{aligned}
$$

and $K_{g}$ is given in eq. (A69). Finally, combining these with the appropriate parton distribution functions gives the $e^{+} p$ cross section of eq. (16). 


\section{A.4 Calculation of $\left\langle k_{T}^{2} \hat{\sigma}_{q, g}\right\rangle$}

The average leptoquark transverse momentum squared is defined by the weighted subprocess cross sections (see eq. (A49))

$$
\begin{aligned}
\left\langle k_{T}^{2} \hat{\sigma}_{q}\right\rangle & =\frac{\lambda^{2} \pi}{4} C_{F} g_{s}^{2} R\left[(1-z)^{2} u(1-u) \psi_{q}(z, u)\right] \\
\left\langle k_{T}^{2} \hat{\sigma}_{g}\right\rangle & =\frac{\lambda^{2} \pi}{4} T_{R} g_{s}^{2} R\left[(1-z)^{2} u(1-u) \psi_{g}(z, u)\right] .
\end{aligned}
$$

The integrations are finite in four dimensions and can be trivially performed. One obtains

$$
\begin{aligned}
\left\langle k_{T}^{2} \hat{\sigma}_{q}\right\rangle & =\frac{\lambda^{2} \pi}{4} C_{F} \frac{\alpha_{S}}{2 \pi} \frac{1}{3}\left[\left(1+z^{2}\right)(1-z)\right], \\
\left\langle k_{T}^{2} \hat{\sigma}_{q}\right\rangle & =\frac{\lambda^{2} \pi}{4} T_{R} \frac{\alpha_{S}}{2 \pi}\left[\frac{1}{2}-3 z+\frac{z^{2}}{2}+2 z^{3}-4 z^{2} \ln z\right] .
\end{aligned}
$$

Folding with the parton distribution functions and dividing by the total cross section gives the result given in eqs. (20,21,22).

\section{References}

[1] H1 Collaboration: C. Adloff et al., DESY preprint 97-24, hep-ex/9702012.

[2] ZEUS Collaboration, J. Breitweg et al., DESY preprint 97-25, hep-ex/9702015(1997).

[3] W. Buchmüller and D. Wyler, Phys. Lett. B177 (1986) 377.

[4] J.C. Pati and A. Salam, Phys. Rev. D10 (1974) 275.

[5] H. Georgi and S.L. Glashow, Phys. Rev. Lett. 32 (1974) 438.

[6] J.L. Butterworth and H. Dreiner, in Proc. of the 2nd HERA Workshop, DESY, Hamburg, Oct. 29-30, 1991; Nucl. Phys. B397 (1993) 7.

[7] S. Davidson, D. Bailey and B.A. Campbell, Z. Phys. C61 (1994) 613.

[8] W. Buchmüller, R. Rückl and D. Wyler, Phys. Lett. B191 (1987) 442.

[9] M. Leurer, Phys. Rev. Lett. 71 (1993) 1324; Phys. Rev. D49 (1994) 333; Phys. Rev. D50 (1994) 536. 
[10] P. Langacker, Phys. Lett. B256 (1991) 277;

Review of Particle Physics, Phys. Rev. D54 (1996) 1.

[11] G. Altarelli, J. Ellis, G.F. Giudice, S. Lola and M.L. Mangano, preprint CERNTH/97-40, hep-ph/9703276;

H. Dreiner and P. Morawitz, preprint hep-ph/9703279.

[12] D0 Collaboration: S. Aid et al., Phys. Rev. Lett. 72 (1994) 965;

'Recent Results from the Search for New Phenomena at D0', presented by Jay Wightman at LaThuile '97: Results and Perspectives in Particle Physics.

[13] J. Blümlein, E. Boos and A. Kryukov, DESY preprint 96-174 (1996).

[14] P. Arnold and C. Wendt, Phys. Rev. D33 (1986) 1873.

[15] G.V Borisov, Y.F. Pirogov and K.R. Rudakov, Z. Phys. C36 (1987) 217.

[16] J. Blümlein and R. Rückl, Phys. Lett. B304 (1993) 337.

[17] J.L. Hewett, T.G. Rizzo, S. Paksava, H.E. Haber and A. Pomarol, Proc. Workshop on Physics at Current Accelerators and the Supercollider (1993).

[18] A.D. Martin, R.G. Roberts and W.J. Stirling, Phys. Lett. B387 (1996) 419.

[19] W. Beenakker, R. Höpker, M. Spira and P.M. Zerwas, preprint CERN-TH/96-215 (1996).

[20] J.L. Hewett and S. Paksava, Phys. Rev. D37 (1988) 3165.

[21] G. Altarelli, J. Ellis, G.F. Giudice, S. Lola and M.L. Mangano, preprint CERNTH/97-40, hep-ph/9703276;

H. Dreiner and P. Morawitz, preprint hep-ph/9703279;

J. Kalinowski, R. Ruckl, H. Spiesberger and P.M. Zerwas, preprint BI-TP-97-07, hepph/9703288;

K.S. Babu, C. Kolda, J. March-Russell and F. Wilczek, preprint hep-ph/9703299;

J.L. Hewett and T.G. Rizzo, preprint SLAC-PUB-7430, hep-ph/9703337;

G.K. Leontaris and J.D. Vergados, preprint IOA-TH-97-004, hep-ph/9703338. 


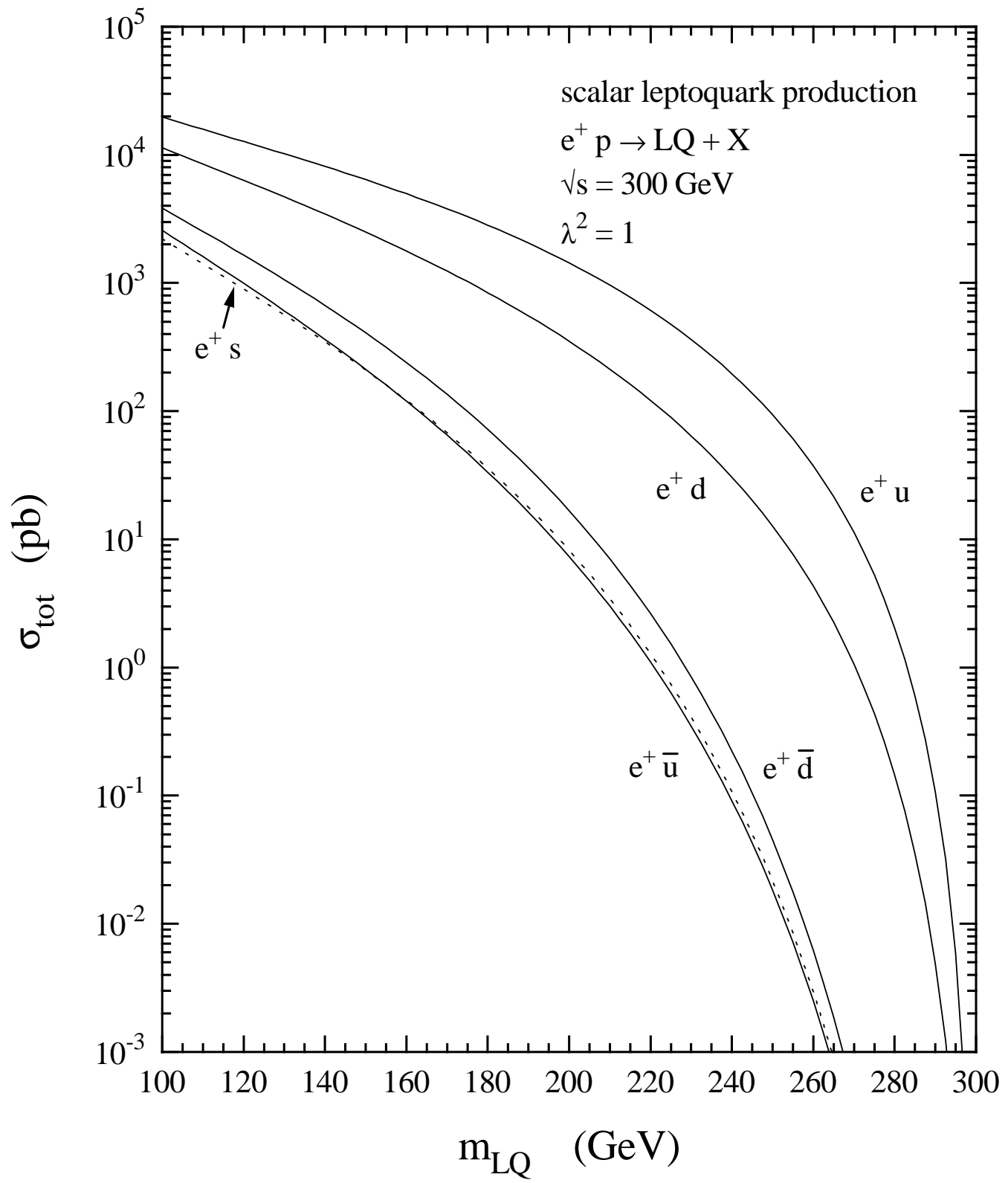

Figure 1: Scalar leptoquark production cross sections in NLO QCD for $e^{+} p$ collisions at $\sqrt{s}=300 \mathrm{GeV}$. 


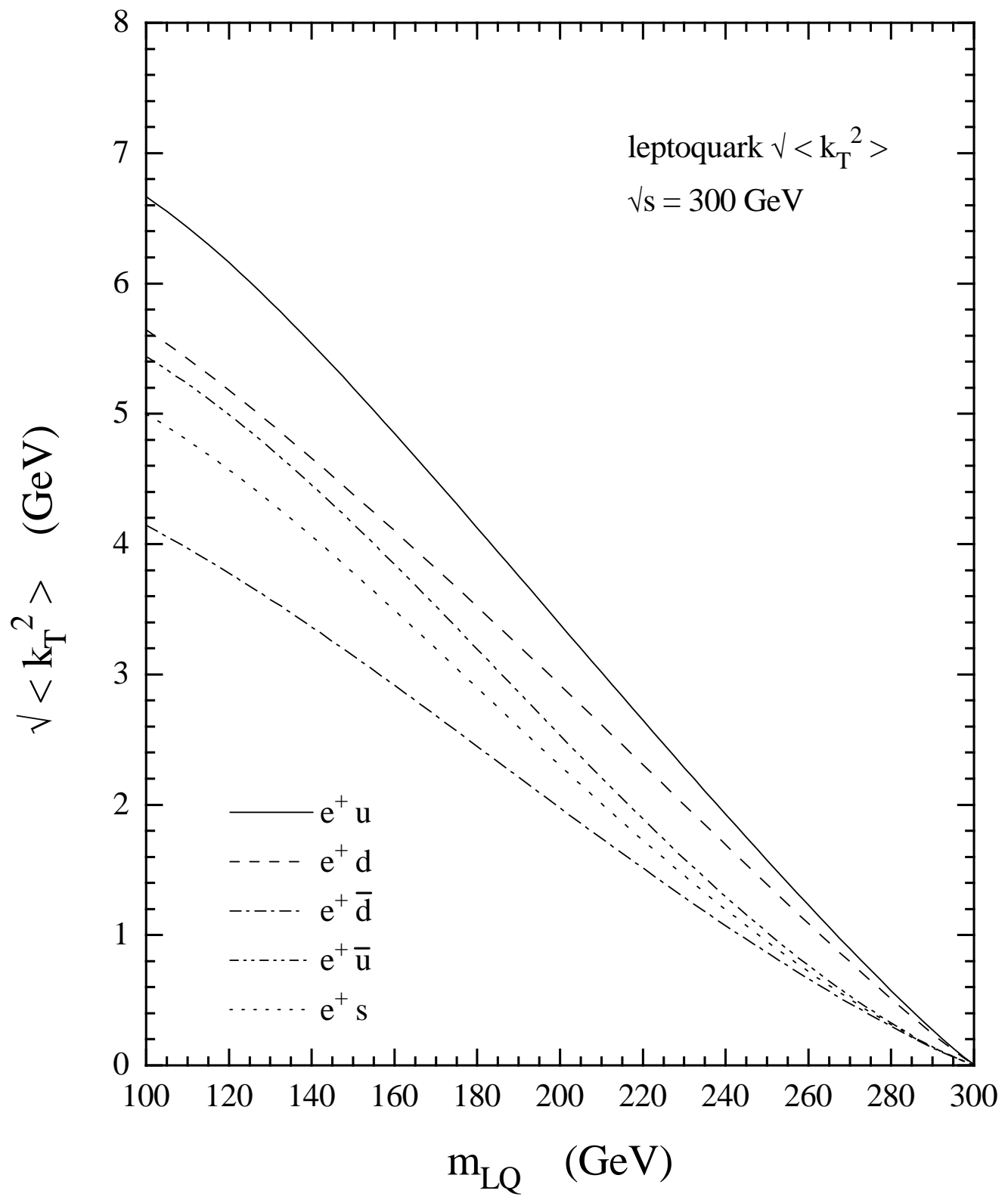

Figure 2: Average scalar leptoquark transverse momentum for $e^{+} p$ collisions at $\sqrt{s}=$ $300 \mathrm{GeV}$. 


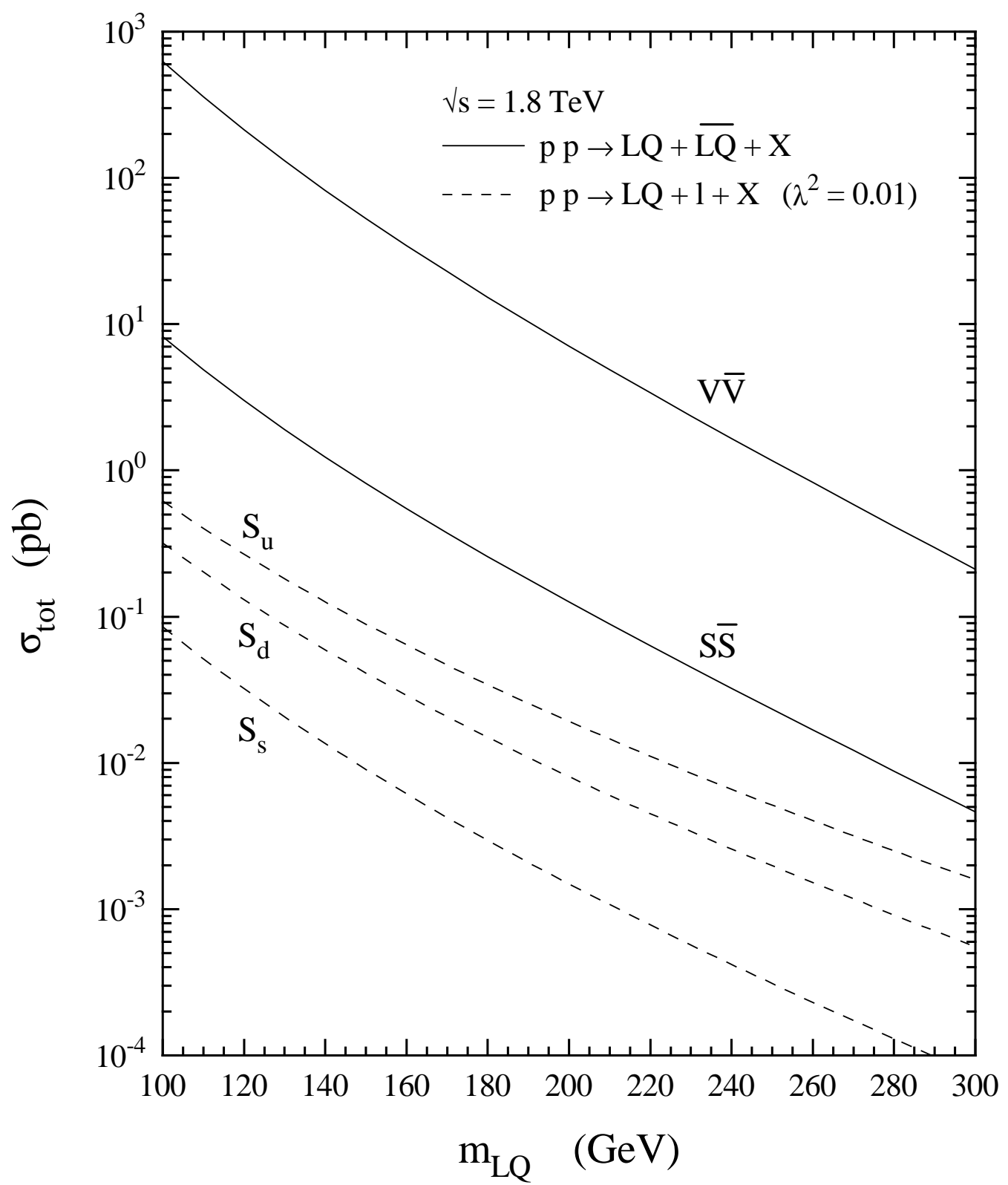

Figure 3: Scalar and vector leptoquark production at the Tevatron $p \bar{p}$ collider. 